\title{
Emerging role of endothelial and inflammatory markers in preeclampsia
}

\author{
Menha Swellam ${ }^{\mathrm{a}, *}$, Nervana Samy ${ }^{\mathrm{a}}$, Susan Abdl Wahab ${ }^{\mathrm{b}}$ and Mohamed Saeed Ibrahim ${ }^{\mathrm{c}}$ \\ ${ }^{a}$ Department of Biochemistry, Genetic Engineering and Biotechnology Research Division, National Research \\ Center, Dokki, Giza, Egypt \\ ${ }^{\mathrm{b}}$ Department of Clinical pathology, Faculty of Medicine, Ain Shams University, Cairo, Egypt \\ ${ }^{\mathrm{c}}$ Department of Obstetrics and Gynecology, Faculty of Medicine, Ain Shams University, Cairo, Egypt
}

\begin{abstract}
Objectives: Endothelial disturbance and excess inflammatory response are pathogenic mechanisms in pre-eclampsia (PE). Authors determine the clinical diagnostic role for thrombomodulin (TM), plasminogen activator inhibitor-1 (PAI-1) as endothelial markers and C-reactive protein (CRP), and interlukin-6 (IL-6) as inflammatory markers when tested independently or in combinations.

Materials and methods: We conducted a retrospective study in a cohort of 185 women grouped as 80 women with PE, 55 normotensive pregnant and 50 healthy non-pregnant. Plasma levels of TM, PAI-1, CRP and IL-6 were examined using enzyme linked immunosorbent assays.

Results: Median levels and the positivity rates for the investigated markers were higher in PE as compared to the other groups $(P<0.0001)$. Using linear regression analysis, the investigated markers were significantly correlated regarding healthy nonpregnant $v s$ PE or normotensive pregnant $v s$ PE. The sensitivity of PAI-1 was the highest $(98 \%)$ among the tested biomarkers. Combination between the investigated markers revealed absolute sensitivity (100\%) and reliable specificity especially when PAI-1 was combined with CRP at $83 \%$ specificity.

Conclusions: Investigated endothelial and inflammatory markers revealed sensitive diagnostic test for PE. However, coupled combination between PAI-1 with CRP showed superior both sensitivity and specificity which represent a promising new approach for detection of PE.
\end{abstract}

Keywords: Pre-eclampsia, thrombomodulin, CRP, IL-6, PAI-1, inflammatory markers, adhesion molecules

Abbreviations: PE; Pre-eclampsia, TM; thrombomodulin, PAI-1; plasminogen activator inhibitor-1, CRP; C-reactive protein, IL-6; interlukin-6.

\section{Introduction}

Preeclampsia (PE) is a multi-system, multi-factorial disease targeted by endothelial damage which precedes the clinical diagnosis; it is estimated to affect $2 \%-10$ of all pregnancies [1] and the fetus depending on the population studied and definitions of PE [2,3] as recent

\footnotetext{
* Corresponding author: Associate Prof. Dr. Menha Swellam, Biochemistry Department, Genetic Engineering and Biotechnology Research Division, National Research Center, Tahrir Street, Dokki, Giza, 12311, Egypt. Tel: +20 23335451; Fax: +20 23370931; E-mail: menha_m_swellam@yahoo.com.
}

studies reported that PE would cluster in families, consistent with a genetic component in the pathophysiology of this disease [3,4]. According to Health Bulletin news, PE accounts for up to $80 \%$ of maternal deaths in the developing world [5].

Data from many studies have indicated that generalized endothelial dysfunction is the cause for the clinical abnormalities in PE [6]. The search for circulating factors mediating this generalized endothelial dysfunction has been the subject of much ongoing research. Among these circulating endothelial markers, thrombomodulin (TM); a natural anticoagulant produced by endothelial cells and bound to their surface [7], and plasminogen 
activator inhibitor-1 (PAI-1); a principle regulator of fibrinolysis [8], are known to play a role in PE.

Specific factors initiating endothelial damage in PE are unknown, although activation of the coagulation system, platelets, and neutrophils are implicated. Normal pregnancy is associated with activation of peripheral blood leukocytes, a response more marked in women with PE. Inflammatory cells are activated in PE and localized to the site of vascular injury. This white cell activation is associated with higher levels of proinflammatory molecules such as C-reactive protein (CRP) [9], and cytokines such as interlukin-6 (IL-6); a multifunctional cytokine that is produced by vascular endothelial cells [10].

The pursuit of safe, reliable, and cost-effective screening tests for the prediction of PE has been the goal of researchers for many decades, with the aim of improving maternal and fetal surveillance, despite the fact that the only current effective treatment remains delivery. To date, however, no specific tests have been proven to be effective and appropriate screening tests for PE. This is partly attributable to the wide range of terminology used for hypertensive disorders in pregnancy, to the varying criteria for the diagnosis of these complex disorders, and to differences in measures of outcome. Many candidates have been examined, but none has proven to be specific and sensitive enough to be of clinical value [11].

The aim for the present study is to evaluate the potential clinical utility of TM, PAI-1, CRP and IL-6 in assessing endothelial damage and prediction of PE when tested independently or in combinations. Moreover, the current study aimed to determine whether circulating endothelial markers (TM and PAI-1) are related to inflammatory markers (CRP and IL-6) in established $\mathrm{PE}$ and which of them best discriminate between PE and normotensive pregnancy.

\section{Materials and methods}

\subsection{Study population and blood sample collection}

The study population, selected from women attending the Obstetrics and Gynecology Department - Ain Shams University Hospital, consisted of 80 women with PE $(27.8 \pm 2.3$, range $22-36$ years $)$ and 55 women with normotensive pregnancies $(27.2 \pm 3.8$, range 21-33 years). Together with a group of 50 healthy non-pregnant women $(26.9 \pm 3.8$, range $22-34$ years $)$ recruited from the hospital laboratory staff. All wom- en gave informed consent to participate in the study, which had been approved by the local Ethics Committee. None of the patients had pre-existing hypertensive disorders or any renal, hepatic, or hematologic diseases, and had received no medication. None of them were smokers.

Pregnant women in the study were cared for by obstetrician, and information about the course of the pregnancy including gestational age, mode of delivery, and any complications of pregnancy, was obtained. Pregnant women were divided into women with PE $(n=80)$ and women with normotensive pregnancy $(n=55)$. Preeclampsia was defined according to the American College of Obstetrics and Gynecology definition (2002) [12], as new-onset hypertension after 20 weeks' gestation, with a diastolic blood pressure of $90 \mathrm{~mm} \mathrm{Hg}$ or higher with concurrent proteinuria of $300 \mathrm{mg} / 24$ hours or greater. Semi-quantitative dipstick tests were used for measurement of proteinuria $(1+$ and $2+$ corresponded to $\geqslant 300 \mathrm{mg} / 24$ hours and $\geqslant$ $500 \mathrm{mg} / 24$ hours, respectively) while normal pregnancy was defined as a pregnancy in which the mother had normal blood pressure $(\leqslant 140 / 90 \mathrm{mmHg})$, absence of proteinuria, and no medical complications.

Blood samples were obtained in the third trimester before any medication was taken. Samples were withdrawn from all individuals and divided equally into two tubes: first tube used to separate the sera for the analysis of CRP and IL-6, while the second tube containing $3.8 \%$ sodium citrate as an anticoagulant used to separate plasma from red blood cells for the determination of TM and PAI-1. For all tube, centrifugation was performed at $3000 \mathrm{rpm}$ for $10 \mathrm{~min}$. and the separated sera or plasma was stored at $-80^{\circ} \mathrm{C}$ until analysis.

\subsection{Measurement of thrombomodulin (TM)}

Plasma thrombomodulin (TM) was measured as indicator of endothelial dysfunction and/or damage with enzyme linked immunosorbent assay using a diagnostic kit supplied by Diagnostica Stago, 9, Rue des Frères Chausson, 92600 Asnières sur Seine, France [13].

\subsection{Measurement of plasminogen activator inhibitor-1 (PAI-1)}

The activity of PAI-1 was measured by commercial two-steps indirect enzymatic assay kit (Spectrolyse/pL PAI kits, Biopool AB). The activity was expressed as Units/ml, where one unit is the amount of PAI-1 that inhibits 1 IU of human single-chain tissue-type plasminogen activator [14]. 
Table 1

Demographic, clinical and laboratory details of the study population

\begin{tabular}{|c|c|c|c|c|c|c|}
\hline \multirow[t]{2}{*}{ Parameters } & \multicolumn{2}{|c|}{ Healthy non-pregnant } & \multicolumn{2}{|c|}{ Healthy normotensive pregnant } & \multicolumn{2}{|c|}{ Preeclampsia } \\
\hline & Median & $95 \% \mathrm{CI}$ & Median & $95 \% \mathrm{CI}$ & Median & $95 \% \mathrm{CI}$ \\
\hline Age (years) & 26 & $25.8-28$ & 27 & $26.1-28.2$ & 28 & $26.9-28.7^{\mathrm{a}}$ \\
\hline $\mathrm{SBP}(\mathrm{mmHg})$ & 120 & $110-120$ & 120 & $110-120$ & $170^{\mathrm{b}, \mathrm{c}, \mathrm{d}}$ & $165-190$ \\
\hline $\mathrm{DBP}(\mathrm{mmHg})$ & 72 & $70-75$ & 72 & $70-75$ & $101^{\mathrm{b}, \mathrm{c}, \mathrm{d}}$ & $94-104$ \\
\hline Parity & - & - & 1 & $0-2$ & 1 & $0-2^{\mathrm{a}}$ \\
\hline Gestational age at delivery (weeks) & - & - & 33.5 & $26-40$ & 31.6 & $24-39^{a}$ \\
\hline $\mathrm{TM}(\mathrm{ng} / \mathrm{ml})$ & 2.2 & $2.1-2.4$ & $2.1^{\mathrm{e}}$ & $2.2-2.5$ & $4.6^{\mathrm{b}, \mathrm{c}, \mathrm{d}}$ & $4.5-4.8$ \\
\hline PAI-1 (U/ml) & 30 & $30-32.5$ & $30.5^{\mathrm{e}}$ & $30-31.9$ & $52^{\mathrm{b}, \mathrm{c}, \mathrm{d}}$ & $50-53$ \\
\hline $\mathrm{CRP}(\mathrm{mg} / \mathrm{L})$ & 2.4 & $2.3-2.7$ & $2.4^{\mathrm{e}}$ & $2.5-3$ & $5.3^{\mathrm{b}, \mathrm{c}, \mathrm{d}}$ & $4.9-5.6$ \\
\hline IL-6 (pg/ml) & 1.1 & $1.1-1.4$ & $1.4^{\mathrm{f}}$ & $1.4-1.68$ & $2.6^{\mathrm{b}, \mathrm{c}, \mathrm{d}}$ & $2.5-3.1$ \\
\hline
\end{tabular}

a Not significant.

${ }^{\mathrm{b}}$ Significant difference at $(P<0.001)$ among investigated groups.

${ }^{\mathrm{c}}$ Significant difference at $(P<0.001)$ compared with healthy non-pregnant.

d Significant difference at $(P<0.001)$ compared with normotensive.

e No significant $(P>0.05)$ between normotensive and healthy non-pregnant groups.

${ }^{\mathrm{f}}$ Significant difference at $(P<0.001)$ between normotensive and healthy non-pregnant groups.

\subsection{Measurement of $C$-reactive protein $(C R P)$}

Determination of serum C-reactive protein (CRP) by a high sensitive immunoassay for measuring human CRP which is a two step sandwich ELISA technique using kit supplied by Diagnostic systems laboratories (DSL-10-42100) Webster, Texas, USA [15].

\subsection{Measurement of interlukin-6 (IL-6)}

Commercially available IL-6 DuoSet Kit enzymelinked immunosorbent assay (ELISA) was used to measure IL-6, following the protocol provided by the manufacturer (BioSource Europe S.A., Rue de industries, 8 B-1400 Nivelles Belgium) [16].

\subsection{Statistical analysis}

All data analyses were performed by means of Statistical Package for the Social Sciences software (SPSS) version 10.0. Results were reported as median and Differences between patients and the control groups were compared with one-way analysis of variance (ANOVA). The threshold value for optimal sensitivity and specificity of TM, PAI-1, CRP, and IL-6 were determined by Receiver Operating Characteristics (ROC) curve, which was constructed by calculating the truepositive fraction (sensitivity $\%$ ) and false-positive fraction (100-specificity \%) of the above-mentioned markers at several cut-off points [17]. The ROC curve can be used to select a cutoff for the diagnostic test that maximizes the sensitivity and minimizes the false-positive rate. The positivity rates were compared by chi-square test. Correlations between variables were studied by linear regression analysis. The level of significance was determined to be less than 0.05 .

\section{Results}

\subsection{Demographic and clinical parameters}

The demographic, clinical and laboratory data of the subjects are shown in Table 1. As expected, the women affected by PE demonstrated significantly higher systolic and diastolic blood pressures $(P<0.001)$ than women with normotensive pregnancies and healthy non-pregnant. Women with PE had significantly higher mean levels of TM, PAI-1, CRP and IL-6 as compared to either normotensive pregnant women or healthy nonpregnant women. Comparing the median levels of normotensive pregnant and healthy non-pregnant groups, a significant difference was only detected in IL-6.

\subsection{Positivity rates of the investigated markers among the three studied groups}

Healthy non-pregnant and normotensive pregnant women were combined in a group and the best cutoff values for investigated parameters were calculated by the ROC curve as $3.65 \mathrm{ng} / \mathrm{ml}$ for TM, $39.6 \mathrm{U} / \mathrm{ml}$ for PAI-1, $3.6 \mathrm{mg} / \mathrm{L}$ for CRP, and $1.42 \mathrm{pg} / \mathrm{ml}$ for IL-6 (Fig. 1). Moreover, calculating the best cutoff values that discriminate between normotensive and PE pregnant women using the ROC curve were nearly the same as follows $(3.65 \mathrm{ng} / \mathrm{ml}$ for TM, $38.9 \mathrm{U} / \mathrm{ml}$ for PAI-1, $3.6 \mathrm{mg} / \mathrm{L}$ for CRP and $1.42 \mathrm{pg} / \mathrm{ml}$ for IL-6). Concerning these cutoff values, all investigated parameters revealed significant difference between the three investigated groups $(P<0.0001)$ (Table 2$)$. 
Table 2

Positivity levels of investigated markers among the different investigated groups

\begin{tabular}{lcccc}
\hline & Thrombomodulin & PAI-1 & CRP & IL-6 \\
\hline Groups & $\geqslant 3.65 \mathrm{ng} / \mathrm{ml}$ & $\geqslant 39.6 \mathrm{U} / \mathrm{ml}$ & $\geqslant 3.6 \mathrm{mg} / \mathrm{L}$ & $\geqslant 1.42 \mathrm{pg} / \mathrm{ml}$ \\
Healthy non-pregnant & $0(0 \%)$ & $0(0 \%)$ & $6(12 \%)$ & $9(18 \%)$ \\
Healthy normotensive pregnant & $5(9.1 \%)$ & $3(5.4 \%)$ & $8(14.5 \%)$ & $25(45.5 \%)$ \\
PE & $65(81.3 \%)^{*}$ & $78(97 \%)^{*}$ & $72(90 \%)^{*}$ & $76(95 \%)^{*}$ \\
\hline
\end{tabular}

*Significant level at $P<0.0001$, Pearson chi-square test.

Table 3

Correlation coefficient between the investigated markers among the different studied groups

\begin{tabular}{|c|c|c|c|c|c|c|}
\hline \multirow[b]{2}{*}{ Markers } & \multicolumn{2}{|c|}{$\begin{array}{l}\text { Healthy non-pregnant } v s \\
\text { normotensive pregnant }\end{array}$} & \multicolumn{2}{|c|}{$\begin{array}{c}\text { Healthy non-pregnant } v s \\
\text { PE }\end{array}$} & \multicolumn{2}{|c|}{$\begin{array}{c}\text { normotensive pregnant } v s \\
\text { PE }\end{array}$} \\
\hline & $\mathrm{R}$ & $P$ & $\mathrm{R}$ & $P$ & $\mathrm{R}$ & $P$ \\
\hline TM vs PAI- 1 & 0.062 & 0.530 & 0.613 & 0.0001 & 0.598 & 0.0001 \\
\hline TM vs CRP & 0.181 & 0.065 & 0.467 & 0.0001 & 0.444 & 0.0001 \\
\hline TM vs IL-6 & 0.131 & 0.182 & 0.566 & 0.0001 & 0.501 & 0.0001 \\
\hline PAI-1 vs CRP & 0.153 & 0.119 & 0.703 & 0.0001 & 0.707 & 0.0001 \\
\hline PAI-1 vs IL-6 & 0.173 & 0.078 & 0.639 & 0.0001 & 0.593 & 0.0001 \\
\hline CRP vs IL-6 & 0.068 & 0.492 & 0.444 & 0.0001 & 0.382 & 0.0001 \\
\hline
\end{tabular}

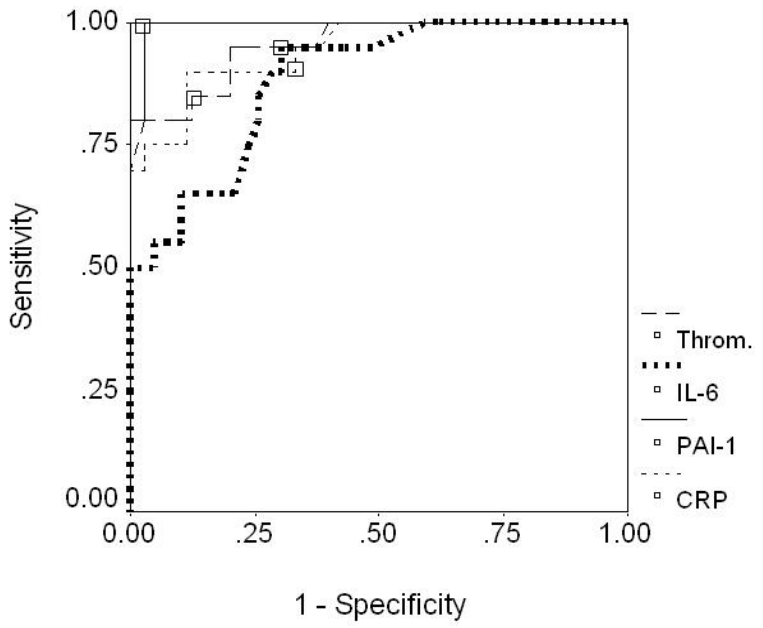

Fig. 1. ROC curve analysis for TM, PAI-1, CRP and IL-6 to calculate the best cutoff points that discriminate between PE and healthy groups. Open squares denote best cutoff points of TM $(3.65 \mathrm{ng} / \mathrm{ml})$ at which sensitivity $=81.3 \%$ and specificity $=95.2 \%$. Area under the curve (AUC) $[\mathrm{SE}]=0.953$ [0.017], 95\% confidence limits range $=0.912-0.979, P<0.001$, and of PAI-1 $(39.6 \mathrm{U} / \mathrm{ml})$ at which sensitivity and specificity $=97 \%$. Area under the curve (AUC) $[\mathrm{SE}]$ $=0.999[0.0001], 95 \%$ confidence limits range $=0.981-1, P<$ 0.0001 , and of CRP $(3.6 \mathrm{mg} / \mathrm{L})$ at which sensitivity $\diamond=90 \%$ and specificity $=86.7 \%$. Area under the curve (AUC) $[\mathrm{SE}]=0.945$ [0.018], 95\% confidence limits range $=0.901-0.973, P<0.0001$, and of IL-6 $(1.42 \mathrm{pg} / \mathrm{ml})$ at which sensitivity $=95 \%$ and specificity $=67.6 \%$. Area under the curve (AUC) $[\mathrm{SE}]=0.884[0.0266], 95 \%$ confidence limits range $=0.829-0.926 P<0.0001$.

\subsection{Correlation between the investigated markers}

Using linear regression analysis, the correlation coefficient between TM, PAI-1, CRP and IL-6 was stud- ied. Significant correlation was detected between the markers $(P<0.0001)$ when consider all investigated groups (Figs 2 a, b, c, d, e, and f), healthy non-pregnant $v s \mathrm{PE}$, and normotensive pregnant vs PE groups, Table 3 .

\subsection{Combined sensitivity and specificity of all investigated markers}

To enable fair comparison for each biomarker among normotensive and PE women, the sensitivities (positivities) of each marker were tested independently or in combination using the cutoff that discriminates normotensive from PE women, as shown in Table 4. Accordingly, both sensitivity and specificity (97\%) of PAI1 were the highest among all investigated markers, followed by IL-6 (95\% for sensitivity and 67.6\% for specificity) then CRP (90\% for sensitivity and $86.7 \%$ for specificity), while TM revealed the lowest sensitivity (81.3\%). Absolute sensitivities and high specificities were reached when CRP was combined with PAI-1 followed by combined CRP with TM (100\% and 83\%; $100 \%$ and $81.9 \% ; 100 \%$ and $79 \%$, respectively).

\section{Discussion}

Pre-eclampsia (PE) remains one of the most serious complications of pregnancy. According to the World Health Organization, PE is a major cause of both maternal and fetal-neonatal morbidity and mortality [18]. Although PE is usually diagnosed in late pregnancy, the 


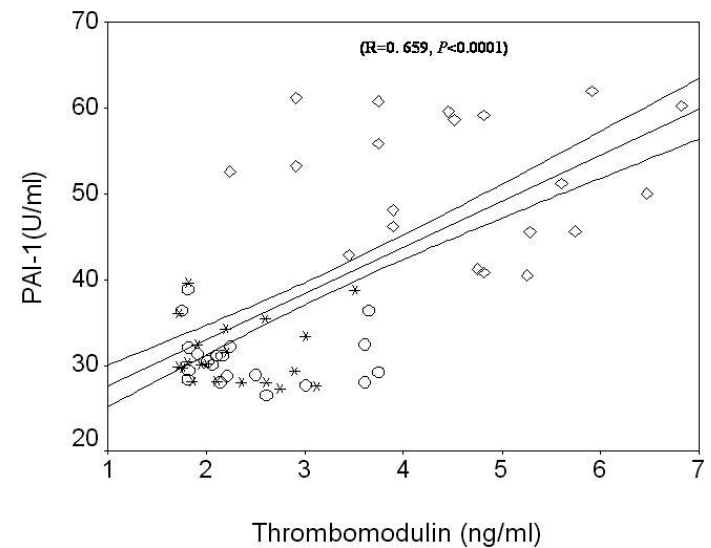

(a)

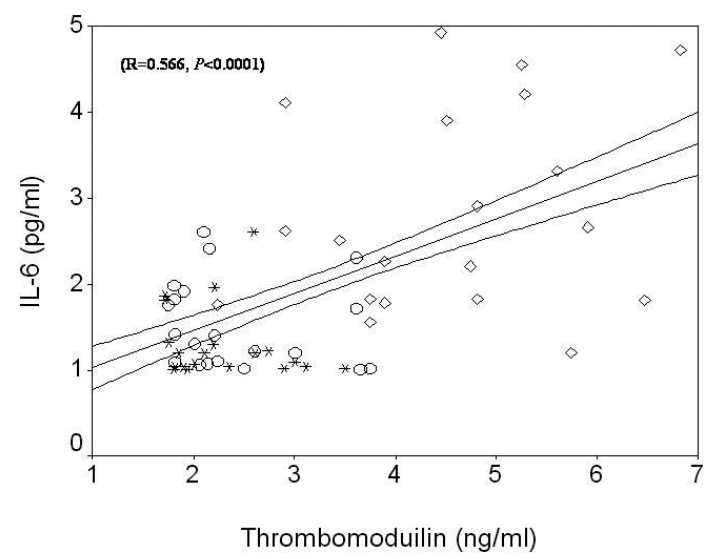

(c)

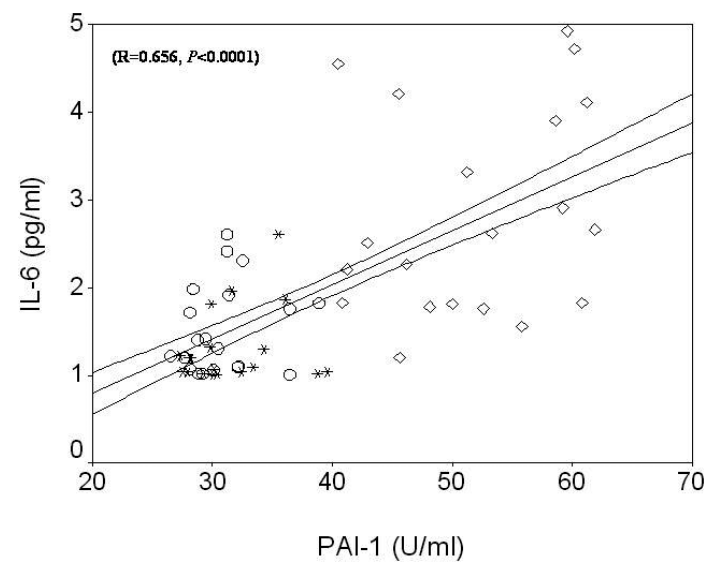

(e)

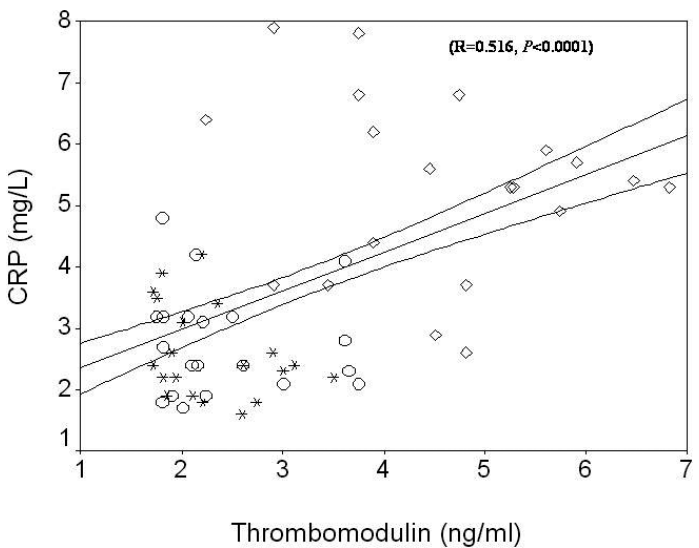

(b)

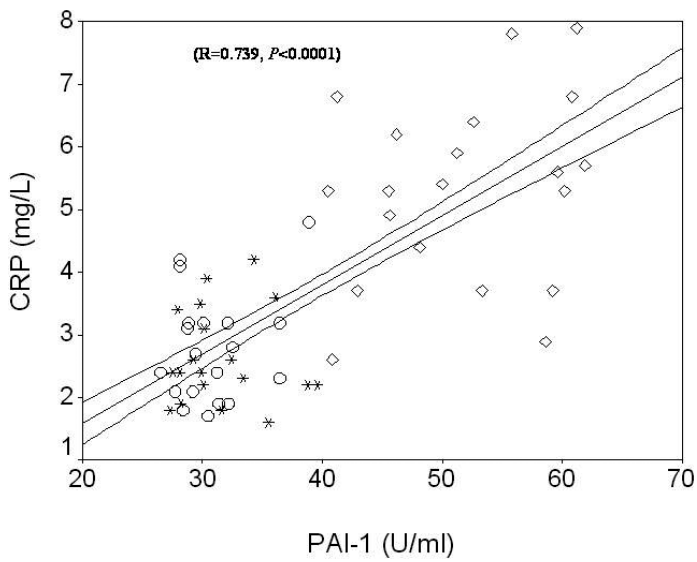

(d)

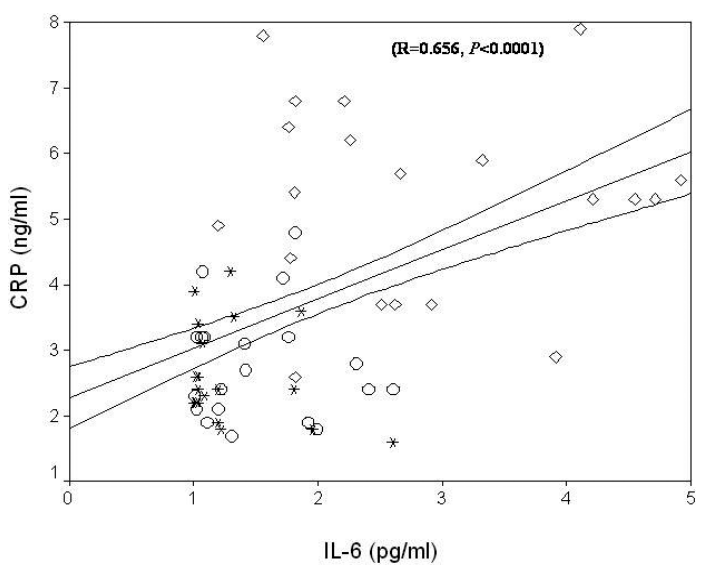

(f)

Fig. 2. Linear regression analysis and $95 \%$ confidence intervals $(95 \% \mathrm{CI})$ between inflammatory and endothelial markers among the different investigated groups (healthy non-pregnant $[\diamond]$; normotensive pregnant $[\bigcirc]$; and PE $[\diamond]$ ). (a) Regression between TM and PAI-1 at 95\%CI = $0.568-0.733$, (b) Regression between TM and CRP at 95\%CI $=0.4027-0.615$, (c) Regression between TM and IL-6 at 95\%CI $=0.4597-0.657$ (d) Regression between PAI-1 and CRP at 95\%CI $=0.6653-0.7979$, (e) Regression between PAI-1 and IL-6 at 95\%CI $=0.5658-0.7315$, and (f) Regression between CRP and IL-6 at $95 \% \mathrm{CI}=0.3515-0.5768$. 
Table 4

Overall sensitivity and specificity, PPV, NPV, accuracy for all investigated markers, when tested independently or combined

\begin{tabular}{lccccc}
\hline & Sensitivity & Specificity & PPV & NPV & Accuracy \\
\hline CRP & 90 & 86.7 & 83.7 & 91.9 & 88 \\
IL-6 & 95 & 67.6 & 69.1 & 94.7 & 79.5 \\
PAI-1 & 97 & 97 & 96.4 & 100 & 98.4 \\
Thrombomodulin & 81.3 & 95.2 & 92.9 & 87 & 89.2 \\
CRP + IL-6 & 100 & 61 & 66.1 & 100 & 77.8 \\
CRP + PAI-1 & 100 & 83 & 82.5 & 100 & 92.3 \\
CRP + Thrombomodulin & 100 & 81.9 & 80.8 & 100 & 89.7 \\
IL-6 + Thrombomodulin & 100 & 63 & 67.2 & 100 & 79 \\
PAI-1 + Thrombomodulin & 100 & 64.8 & 68.4 & 100 & 96.2 \\
CRP + IL-6 + PAI-1 & 100 & 59 & 65 & 100 & 76.8 \\
CRP + IL-6 + Thrombomodulin & 100 & 56.2 & 63.5 & 100 & 75 \\
CRP + PAI-1 + Thrombomodulin & 100 & 79 & 78.4 & 100 & 88 \\
IL-6 + PAI-1 + Thrombomodulin & 100 & 60 & 65.6 & 100 & 77.9 \\
CRP + IL-6 + PAI-1 + Thrombomodulin & 100 & 53 & 62 & 100 & 73.5 \\
\hline
\end{tabular}

roots of the disease are present in the first half of pregnancy. Thus insight to assess the clinical implication of the circulating markers in PE for early diagnosing and screening of PE is highly recommended.

The glomerular and pathophysiological changes of PE, especially those present before clinically evident disease, suggest that altered endothelial function is responsible for many of the changes in the syndrome [19]. Our study demonstrated an elevated $(P<0.001)$ median levels and positivity rates of endothelial markers as compared to normotensive pregnant or healthy nonpregnant women. Previously, it was reported that increased TM in plasma reflects the degree of endothelial damage that is caused as a result of hypertension [20]. Moreover, the increased levels of PAI-1, a member regulating fibrinolysis [21], in PE may be attributed to the hypothesis that hypoxia may be responsible for increased syncytial PAI-1 expression noted in PE, which is suggested to promote increased intervillous fibrin deposition that is observed in these pregnancies [22]. PE is secondary to the interactions of reduced placental perfusion with diverse maternal factors that alter endothelial function. The maternal contribution is from factors that antedate pregnancy and are influenced by the usual metabolic adaptations of pregnancy. The endothelium and other targets for the effects of these interactions are more sensitive to insults during pregnancy because of activation of the inflammatory cascade as a normal part of pregnancy [19].

Of the inflammatory markers, we have assessed CRP and IL-6 in the three investigated groups. Accordingly, our results revealed positive significant increase both median and positivity rates in PE than normotensive pregnant women and healthy non-pregnant ones. Plasma from women with PE can activate vascular endothe- lial cells through an $\mathrm{NK}-{ }_{\kappa} \mathrm{B}-$ mediated mechanism, these cells could be a potential source of increased circulating IL-6 that seen in this disease [10]. Production of CRP is stimulated by the release of proinflammatory cytokines including interleukin- 6 which may explain the significant increase of CRP levels reported in this study.

Also, it has been suggested by Freeman and his colleagues [23] that PE is attributable to an excessive maternal inflammatory response to pregnancy secondary to a combination of placental factors and maternal factors related to phenotype and genotype. This inflammatory response contributes to the wider syndrome of endothelial dysfunction and thrombotic and metabolic disturbances seen in PE. In the present study we have examined the correlation between all of the investigated endothelial and inflammatory markers in the three investigated groups. Interestingly, significant correlations were detected when comparing healthy nonpregnant or normotensive pregnant women $v s$ PE which signifies the clinical impact of the investigated markers. These findings may support the hypothesis that endothelial disfunction, activation or damage in women with PE may contribute to excess inflammatory production. A prospective longitudinal investigation to further study the various circulating endothelial and inflammatory markers to more reliably identify women at high risk of PE is in progress.

Preeclampsia is not a single disorder but rather a syndrome [24]. It has become increasingly clear that it is unlikely a single test will be able to predict all forms of PE early in pregnancy. Hence a new strategy is needed to focus on the identification of women at risk of developing PE, based on combination of biochemical markers. To the best of our knowledge, this is the 
first study to specifically investigate all these markers concurrently in PE and study their combined overall sensitivity and specificity, PPV, NPV as early, reliable and safe diagnostic and screening tests. Our results showed that the sensitivity for early detection of PE was absolute when the investigated markers were combined. However the best combination was observed between CRP and PAI-1, followed by CRP and TM as regard to their sensitivity and specificity $(100 \%, 83 \%$, and $100 \%$, $81.9 \%$, respectively).

Taken together, these findings suggest that a positive correlation was detected between inflammatory and endothelial markers. Moreover, combined testing of CRP and PAI-1 may improve the sensitivity and specificity for diagnosing and screening of PE. From a diagnostic standpoint, although we believe that the discovery of soluble endothelial and inflammatory markers used either alone or in combination with other markers offers tremendous promise in the diagnosis and screening of $\mathrm{PE}$, we recognize that the limited number of patients in this study comprise just a pilot study, and future studies based on large number of patients to confirm these findings are highly recommended. Once this is achieved, a safe, reliable, and cost-effective screening tests may become available for routine clinical use for diagnosing and screening PE.

\section{References}

[1] J.M. Roberts, R.N. Taylor, T.J. Musci, G.M. Rodgers, C.A. Hubel and M.K. McLaughlin, Preeclampsia: an endothelial cell disorder, Am J Obstet Gynecol 161 (1989), 1200-1204.

[2] C.W.G. Redman and I.L. Sargent, The pathogenesis of preeclampsia, Gynécol Obstét Fertil 29 (2001), 518-522.

[3] K. Duckitt and D. Harrington, Risk factors for pre-eclampsia at antenatal blooking: systemic review of controlled studies, BMJ 330 (2005), 565-573.

[4] D.B. Carr, M. Epplein, C.O. Johnson, T.R. Easterling and C.W. Critchlow, A sister's risk: Family history as a predictor of preeclampsia, Am J Obstet and Gynecol 193 (2005), 965-972.

[5] L.M. Bodnar, Low vitamin D level early in pregnancy increases preeclampsia risk 500 percent, Health Bulltein 9 (2007), 3517-3120.

[6] C. Lam, K.-H. Lim and S.A. Karumanchi, Circulating Angiogenic Factors in the Pathogenesis and Prediction of Preeclampsia, Hypertension 46 (2005), 1077-1085.

[7] A. Remkova, E. Kovacova, M. Prýkazska and H. Kratochvýl'ova, Thrombomodulin as a marker of endothelium damage in some clinical conditions, Europ J Internal Medicine 11 (2000), 79-84.

[8] Y. Hashimoto, A. Kobayashi, N. Yamazaki, Y. Sugawara, Y. Takada and A. Takada, Relationship between age and plasma t-PA, PA-inhibitor, and PA activity, Thromb Res 46 (1987), 625-633.

[9] W. Pitiphat, M.W. Gillman, K.J. Joshipura, P.L. Williams,
C.W. Douglass and J.W. Rich-Edwards, Plasma C-Reactive Protein in early pregnancy and preterm delivery, Am J Epidemiol 162 (2005), 1108-1113.

[10] P. Takacs, K.L. Green, A. Nikaeo and S.W. Kauma, Increased vascular endothelial cell production of interleukin- 6 in severe preeclampsia, Am J Obstet and Gynecol 188 (2003), 740-744.

[11] K.H. Lim and M.M. Watkins, Preeclampsia, eclampsia, and hypertension, in: Current Clinical Pathology: Handbook of Clinical Laboratory Testing During Pregnancy, A.M. Gronowski, ed., Totowa, NJ: Humana Press; 2004, pp/ 391400.

[12] A.C.O.G. Committee on Obstetric Practice. ACOG practice bulletin. Diagnosis and management of preeclampsia and eclampsia. Number 33, January 2002. American College of Obstetricians and Gynecologists, Int J Gynaecol Obstet 77 (2002), 67-75.

[13] R.M. Loreth, C.H. Seus, H.H. Berger and F.W. Albert, Plasma thrombomodulin concentrations in healthy persons depend on age and sex, Annals of Hematology 74(Suppl II) (1997), A89A94.

[14] E. Eriksson, M. Ranby, E. Gyzander and B. Risberg, Determination of plasminogen activator inhibitor in plasma using t-PA and a chromogenic single-point poly-D-lysine stimulated assay, Thromb Res 50 (1988), 91-101.

[15] N. Rifai, R. Tracy and P. Ridker, Clinical efficacy of an automated high-sensitivity $\mathrm{C}$-reactive protein assay, Clin Chem $\mathbf{4 5}$ (1999), 2136-2141.

[16] O. Le Moineo, Interleukin-6: an early marker of bacterial infection in decompensated cirrhosis. J. Hepatology 20 (1994), 819-824.

[17] Zweig $\mathrm{MH}$ and Campbell G Receiver-operating characteristic (ROC) plots: a fundamental evaluation tool in clinical medicine, Clin Chem 39 (1993), 561-577.

[18] N. Ilhan, N. lhan and M. Simsek, The changes of trace elements, malondialdehyde levels and superoxide dismutase activities in pregnancy with or without preeclampsia, Clin Biochem 35 (2002), 393-397.

[19] J.M. Roberts and K.Y. Lain, Recent Insights into the Pathogenesis of Pre-eclampsia, Placenta 23 (2002), 359-372.

[20] S.K. Nadar, E.A. Yemeni, A.D. Blann and G.Y.H. Lip, Thrombomodulin, von Willbrand factor and E-selectin as plasma markers of endothelial damage/dysfunction and activation in pregnancy induced hypertension, Thromb Res 113 (2004), 123-128.

[21] S. Guller, Y. Ma, A. Malek, S.D. Santo and H. Schneider, Differential Release of Plasminogen Activator Inhibitors (PAIs) During Dual Perfusion of Human Placenta: Implications in Preeclampsia, Placenta 28 (2007), 278-285.

[22] A. Estelles, J. Gilabert, M. Keeton, Y. Eguchi, J. Aznar, S. Grancha et al., Altered expression of plasminogen activator inhibitor type 1 in placentas from pregnant women with preeclampsia and/or intrauterine fetal growth retardation, Blood 84 (1994), 143-145.

[23] D.J. Freeman, F. McManus, E.N. Brown, L. Cherry, J. Norrie, J.E. Ramsay, P. Clark, I.D. Walker, N. Sattar and I.A. Greer, Short- and Long-Term Changes in Plasma Inflammatory Markers Associated With Preeclampsia, Hypertension 43 (2004), 708-714.

[24] N.G. Than, R. Remero, R. Hillermann, V. Cozzi, G. Nie and B. Huppertz, Prediction of preeclampsia - A workshop report, Placenta 22 (2008), S83-S85. 


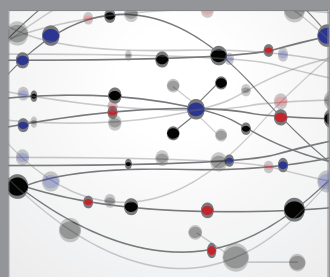

The Scientific World Journal
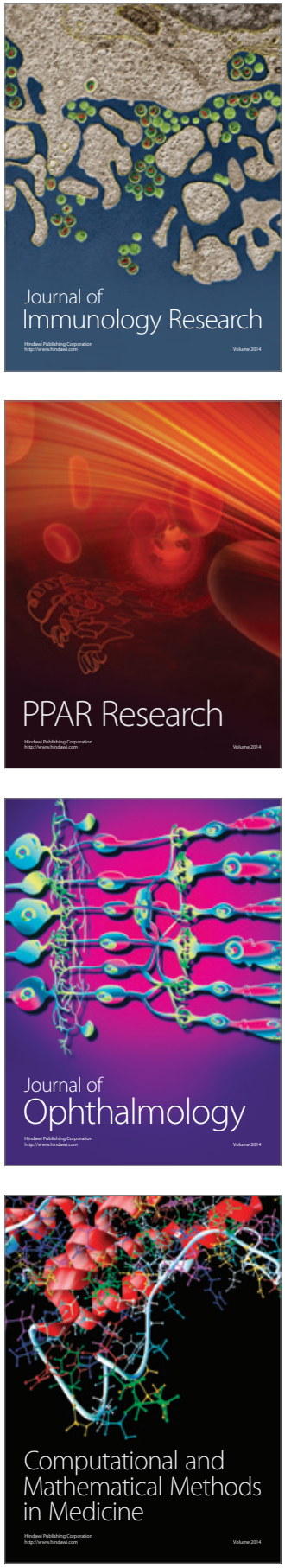

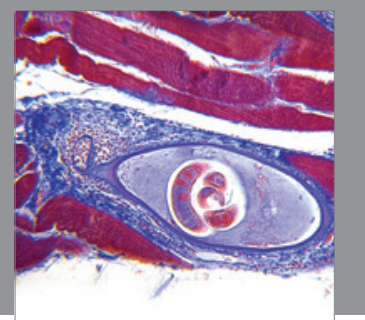

Gastroenterology

Research and Practice
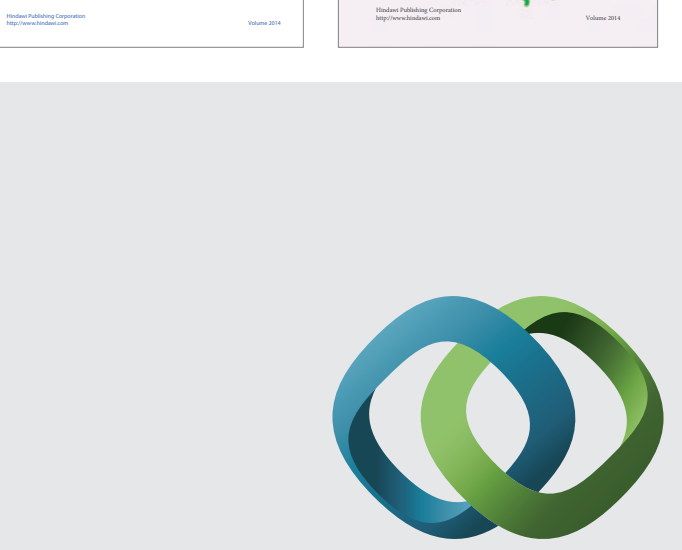

\section{Hindawi}

Submit your manuscripts at

http://www.hindawi.com
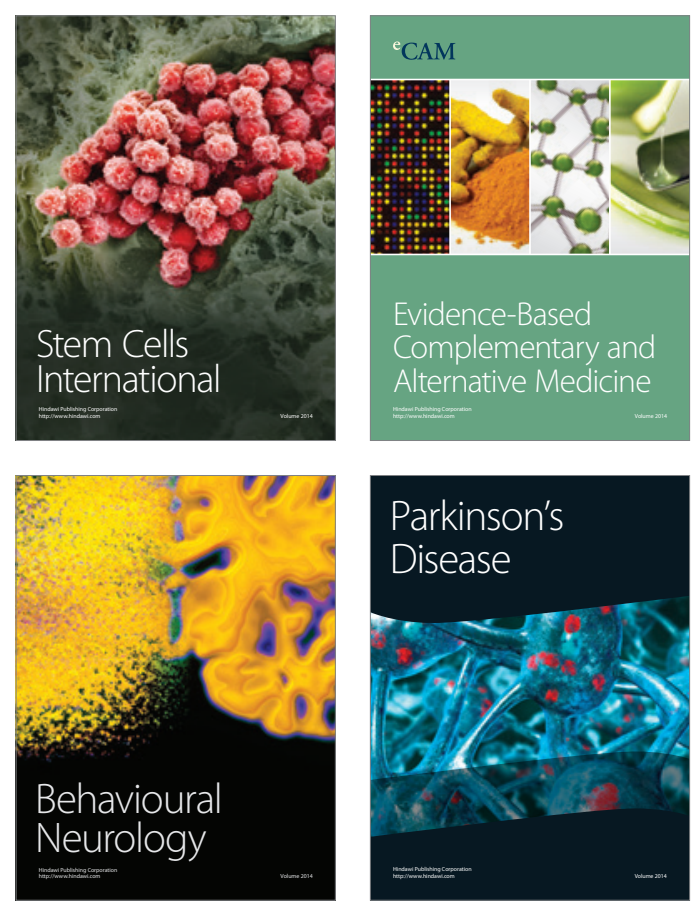

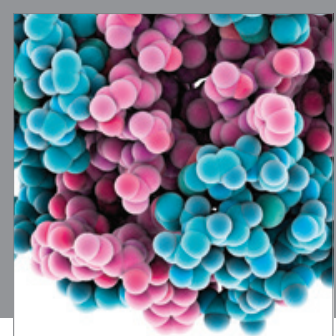

Journal of
Diabetes Research

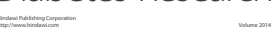

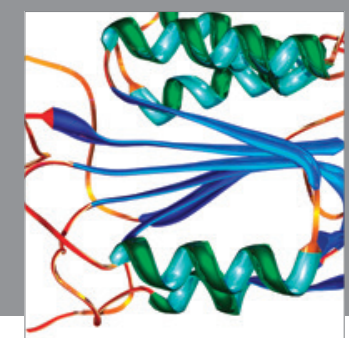

Disease Markers
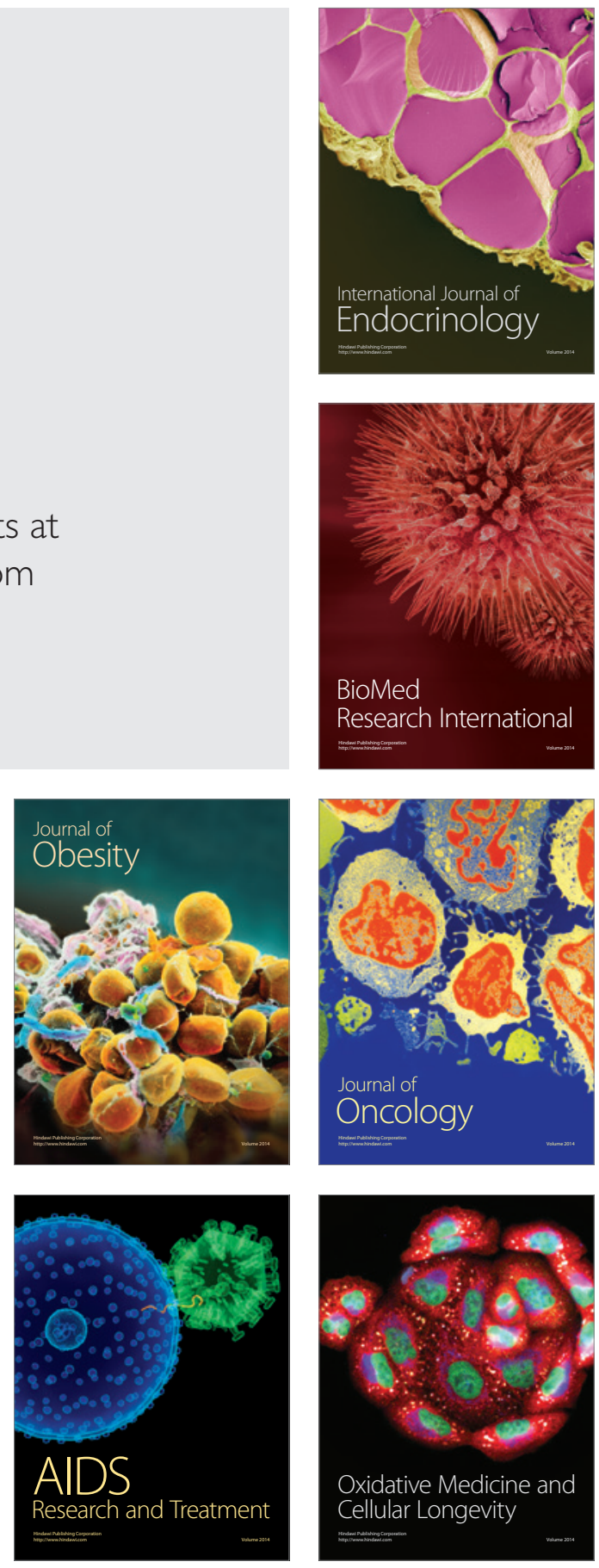